Psychotherapeut 2019 $64: 177-179$

https://doi.org/10.1007/s00278-019-0355-z

(c) Springer Medizin Verlag GmbH, ein Teil von Springer Nature 2019

\author{
Anja Hilbert ${ }^{1}$ Ann.-Christine Ehlis ${ }^{2}$ \\ ${ }^{1}$ Integriertes Forschungs- und Behandlungszentrum AdipositasErkrankungen, Medizinische Psychologie \\ und Medizinische Soziologie und Psychosomatische Medizin und Psychotherapie, Universitätsmedizin \\ Leipzig, Leipzig, Deutschland \\ ${ }^{2}$ Klinik für Psychiatrie und Psychotherapie, Universität Tübingen, Tübingen, Deutschland
}

\title{
Neurowissenschaftlich fundierte Psychotherapie
}

Die neurowissenschaftliche Forschung hat in den vergangenen Jahren vermehrt Einblicke in neurokognitive und -physiologische Auffälligkeiten gewährt, die für psychische Störungen kennzeichnend sind. So wurde beispielsweise auf der Grundlage von neuropsychologischen Tests gefunden, dass Essstörungen wie die Binge-Eating-Störung durch neurokognitive Dysfunktionen gekennzeichnet sind, die in Beeinträchtigungen in der inhibitorischen Kontrolle und im Belohnungssystem bestehen, und zwar besonders bei der Verarbeitung von störungsrelevantem Material wie Nahrungsreizen (Kittel et al. 2015). Bildgebungsstudien, hauptsächlich auf Basis funktioneller Magnetresonanztomographie (fMRT), dokumentierten zudem eine differenzielle Aktivierung bestimmter Hirnregionen in der Verarbeitung von Nahrungsreizen, beispielsweise eine Hypoaktivität in präfrontalen Kontrollnetzwerken und eine Hyperaktivität im medialen orbitofrontalen Kortex. Solche Befunde legen ein verändertes Störungsverständnis nahe: Im Falle der Binge-Eating-Störung wäre in einem aktualisierten Störungsmodell die Aufrechterhaltung der Essanfälle durch inhibitorische Kontrolldefizite und eine erhöhte Belohnungssensitivität $\mathrm{zu}$ erklären, zusätzlich zu den „klassischen“ Aufrechterhaltungsfaktoren eines negativen Körperbildes und einer negativen Affektivität, sodass entsprechende neurowissenschaftlich fundierte Behandlungsempfehlungen abgeleitet werden können (Hilbert 2019). Denn in „klassischen“ psychotherapeutischen Ansätzen wie der kognitiven Verhaltenstherapie werden diese Faktoren zumeist nicht direkt adressiert.

Aufbauend auf neuropsychologischen und -physiologischen Befunden sowie den Ergebnissen struktureller und funktioneller Bildgebung wurden neurowissenschaftlich fundierte Therapieansätze entwickelt, die darauf abzielen, störungsrelevante neurokognitive und -physiologische Prozesse direkt zu verändern, beispielsweise das Neurofeedback für die Binge-Eating-Störung, das die inhibitorische Kontrolle in Bezug auf Nahrungsreize $\mathrm{zu}$ verbessern sucht (Blume et al. 2017). Mit der Entwicklung neurowissenschaftlich fundierter Therapieansätze ist die Erwartung verbunden, die Wirksamkeit von Psychotherapie weiter zu steigern, insbesondere wenn beide Ansätze direkt oder indirekt miteinander kombiniert werden. Obwohl für viele psychische Störungen wirksame evidenzbasierte Psychotherapie vorliegt, ist weiterer Verbesserungsbedarf gegeben.

Das Sonderheft der Zeitschrift Psychotherapeut gibt einen Überblick über verschiedene neurowissenschaftlich fundierte Therapieansätze. Aktuelle Studienergebnisse zu neurokognitiven Trainings, zum Neurofeedback sowie zur nichtinvasiven transkraniellen Hirnstimulation bei verschiedenen psychischen Störungen werden vorgestellt und in Bezug auf ihren Einsatz in der Psychotherapie diskutiert. Das vorliegende Sonderheft der Zeitschrift Psychotherapeut behandelt somit die Frage, ob neurowissenschaftlich fundierte Therapieansätze zukünftig eine wirksame Option in der Psychotherapie darstellen können.
Die „Cognitive Bias Modification“ bezeichnet eine relativ stark beforschte Familie von Interventionen, die darauf abzielen, kognitive Verzerrungen direkt zu behandeln, indem die Patienten anhand von neuropsychologischen Aufgaben trainiert werden, deren Kontingenzen bestimmte Verarbeitungsmuster selektiv begünstigen (MacLeod und Mathews 2012). Während aktuelle Übersichtsarbeiten der monotherapeutischen „Cognitive Bias Modification“ zur Veränderung von Aufmerksamkeitsund Interpretationsbiases zumindest kurzfristig eine mögliche Wirksamkeit für verschiedene psychopathologische Symptome bescheinigen (z. B. Jones und Sharpe 2017), gehen Tendolkar et al. der klinisch relevanten Frage nach, inwieweit die "Cognitive Bias Modification“ als Zusatzintervention die Effekte von Standardpsychotherapie bei Depression verbessern kann.

Neurofeedback ist eine spezielle Anwendung des Biofeedbacks, bei der bestimmte Aspekte der Hirnaktivität in Echtzeit erfasst und verarbeitet werden, um sie dem "Lerner" - meist in Form eines einfachen visuellen Reizes - unmittelbar zurückzumelden, wobei dieser wiederum versuchen soll, den entsprechenden Parameter aktiv zu regulieren. Gelingt ihm die gewünschte Regulation (z.B. die Aktivität in einem bestimmten Frequenzbereich des Elektroenzephalogramms zu erhöhen), wird dies über visuelle und verbale FeedbackSignale (z.B. Lob durch den Trainer) positiv verstärkt. Hierbei sollen durch Mechanismen der klassischen und operanten Konditionierung eine verbesserte 
Selbstregulation der eigenen neuronalen Aktivität erreicht und schließlich dysfunktionale Hirnaktivierungsmuster „normalisiert“ werden. In der neurowissenschaftlichen Forschung wurde der Einsatz von Neurofeedback bisher v.a. für die Aufmerksamkeitsdefizit-/ Hyperaktivitätsstörung (ADHS) im Rahmen kontrollierter Studien überprüft; der Großteil der Trainings basiert auf der Methode der Elektroenzephalographie (EEG; Arns et al. 2014). In einer praxisorientierten Übersicht stellt Enriquez-Geppert den derzeitigen Stand der Forschung zu EEG-basiertem Neurofeedback dar, diskutiert seine Effizienz und stellt ein neueres Protokoll vor, nämlich ein frontomediales Theta-Training, das auf eine Verbesserung exekutiver Funktionen abzielt und somit potenziell weitreichende klinische Bedeutung hat.

Barth und Ehlis präsentieren eine Übersicht über Neurofeedback speziell zur Behandlung der adulten ADHS, für die - nach erfolgreicher Anwendung im Kindes- und Jugendalter - erst in den letzten Jahren regelmäßig entsprechende Studien durchgeführt wurden. Auch hier stehen bislang EEG-basierte Protokolle im Vordergrund, während sich Neurofeedback-Trainings, basierend auf anderen Modalitäten (z.B. funktionelle Nahinfrarotspektroskopie [fNIRS]; Hudak et al. 2017, 2018; Ehlis et al. 2018) und funktionelle Magnetresonanztomographie in Echtzeit (rtfMRT; Zilverstand et al. 2017) derzeit noch in der Entwicklung befinden. Im Rahmen einer Pilotstudie stellen Storchak et al. speziell die Entwicklung eines fNIRS-basierten Trainingsprotokolls vor, das auf eine Verringerung akustisch-verbaler Halluzinationen bei Patienten mit schizophrenen Erkrankungen abzielt. Karch et al. geben schließlich eine narrative Übersicht über den Einsatz der „Real-time“(rt)fMRT bei psychischen Erkrankungen, speziell mit Blick auf die Modulation emotionaler und motivationaler Prozesse.

Verfahren der nichtinvasiven Hirnstimulation ermöglichen die gezielte Modulation neuronaler Aktivität; im Rahmen entsprechender Protokolle ist entweder eine vorübergehende Inhibition oder Fazilitation (d.h. „Aktivierung“) kortikalen Gewebes möglich (Ehlis et al. 2016). Da- bei werden - im Unterschied zu Neurofeedback-Trainings - externe Reize, häufig in Form von Magnetpulsen (repetitive transkranielle Magnetstimulation, rTMS) oder Strom (z.B. transkranielle Gleichstromstimulation [„transcranial direct current stimulation“, tDCS]), verwendet. Herrmann et al. geben in einem systematischen Review Aufschluss über zusätzliche Effekte transkranieller Magnetstimulation, die zur Behandlung von Angsterkrankungen - entweder kurz vor oder während expositionsbasierter Verhaltenstherapie - zum Einsatz kam. Fink und Exner integrieren weiterhin den Forschungsstand zur nichtinvasiven Hirnstimulation (rTMS und tDCS) zur Behandlung von Zwangsstörungen mit insgesamt vielversprechenden Ergebnissen.

In der Zusammenschau zeigt sich für verschiedene Störungsbilder zwar ein deutlicher Wissenszuwachs zu neurowissenschaftlich orientierten Interventionen. Vielfach unbeantwortet bleiben jedoch Fragen hinsichtlich: der Operationalisierung der Trainingsprotokolle (z. B. Bestimmung der Zielregion, Intensität); des Auftretens von Nebenwirkungen; der Absicherung der „wahren“ Effekte eines Trainings im Vergleich zu Placebobedingungen; der vergleichenden Wirksamkeit untereinander und hinsichtlich „klassischer" Psychotherapie. Weitgehend unklar ist zudem, welche Patienten von neurowissenschaftlich orientierten Verfahren besonders gut profitieren. Eine groß angelegte Studie von Drysdale et al. (2017) zeigte beispielsweise, dass Patienten mit Depression sich anhand unterschiedlicher Auffälligkeiten in der fMRT-basierten Konnektivität in limbischen und frontostriatalen Netzwerken in vier neurophysiologische Subtypen aufteilen lassen, die zugleich den Behandlungserfolg von nichtinvasiver transkranieller Magnetstimulation vorhersagten. Ähnliche Befunde deuten sich auch für (erwachsene) Patienten mit ADHS an, die sich im Rahmen einer ClusterAnalyse in diskrete neurophysiologische „Biotypen“ - mit jeweils spezifischen Mustern funktioneller Auffälligkeiten unterteilen ließen (Barth et al. 2018). Unklar bleiben des Weiteren häufig die klinisch-praktische Anwendbarkeit und der potenzielle additive Nutzen von neurowissenschaftlich orientierten Interventionen als Zusatz zu „klassischer“ Psychotherapie. Am Weitesten gediehen scheint der Bereich des adjuvanten EEG-Neurofeedbacks für die ADHS bei Kindern, das 2018 als ergänzende Behandlungsoption Eingang in die überarbeitete S3-Leitlinie Aufmerksamkeits-/ Hyperaktivitätsstörung im Kindes-, Jugend- und Erwachsenenalter (Arbeitsgemeinschaft der Wissenschaftlichen Medizinischen Fachgesellschaften 2018) gefunden hat.

\section{Korrespondenzadresse}

\section{Prof. Dr. Anja Hilbert}

Integriertes Forschungs- und Behandlungszentrum AdipositasErkrankungen, Medizinische Psychologie und Medizinische Soziologie und Psychosomatische Medizin und Psychotherapie, Universitätsmedizin Leipzig Philipp-Rosenthal-Str. 27, 04103 Leipzig, Deutschland

anja.hilbert@medizin.uni-leipzig.de

Danksagung. Die Autorinnen danken Frederike Obereigner für ihre editorische Unterstützung des Manuskripts.

Förderung. A. Hilbert wird gefördert durch das Bundesministerium für Bildung und Forschung, FKZ 01E01501. A.-C. Ehlis wird gefördert durch das IZKF Tübingen (Nachwuchsgruppe 2115-0-0).

Interessenkonflikt. A. Hilbert und A.-C. Ehlis geben an, dass kein Interessenkonflikt besteht.

\section{Literatur}

Arbeitsgemeinschaft der Wissenschaftlichen Medizinischen Fachgesellschaften (2018) S3 Leitlinie Aufmerksamkeitsdefizit-/ Hyperaktivitätsstörung im Kindes-, Jugend- und Erwachsenenalter. https://www. awmf.org/uploads/tx_szleitlinien/028-045I_ S3_ADHS_2018-06.pdf. Zugegriffen: 11. März 2019

Arns M, Heinrich H, Strehl U (2014) Evaluation of neurofeedback in ADHD: the long and winding road. Biol Psychol 95:108-115

Barth B, Mayer-Carius K, Strehl U, Kelava A, Häußinger FB et al (2018) Identification of neurophysiological biotypes in attention deficit hyperactivity disorder. Psychiatry Clin Neurosci 72:836-848

Blume M, Schmidt R, Hilbert A (2017) Biofeedback: Eine wirksame Ergänzung in der Behandlung von Ess- und Gewichtsstörungen? Psychotherapeut 62:204-211

Drysdale AT, GrosenickL, Downar J, DunlopK, Mansouri F et al (2017) Resting-state connectivity biomarkers define neurophysiological subtypes of depression. Nat Med 23:28-38

Ehlis AC, Barth B, Hudak J, Storchak H, Weber L et al (2018) Near-Infrared Spectroscopy as a new tool for neurofeedback training: applications in 
psychiatry and methodological considerations. Jpn Psychol Res 60:225-241

Ehlis AC, Haeussinger FB, Gastel A, Fallgatter AJ, Plewnia C (2016) Task-dependent and polarityspecific effects of prefrontal transcranial direct current stimulation on cortical activation during word fluency. Neuroimage 140:134-140

Hilbert A (2019) Binge-eating disorder. Psychiatr Clin North Am 42:33-43

Hudak J, Blume F, Dresler T, Haeussinger FB, Renner TJ et al (2017) Near-infrared spectroscopy-based frontal lobe neurofeedback integrated in virtual reality modulates brain and behavior in highly impulsive adults. Front Hum Neurosci 11:425

Hudak J, Rosenbaum D, Barth B, Fallgatter AJ, Ehlis AC (2018) Functionally disconnected: a look at how study design influences neurofeedback data and mechanisms in attention-deficit/hyperactivity disorder. PLoS ONE 13:e200931

Jones EB, Sharpe L (2017) Cognitive bias modification: a review of meta-analyses. J Affect Disord 223:175-183

Kittel R, Brauhardt A, Hilbert A (2015) Cognitive and emotional functioning in binge-eating disorder: a systematic review. Int JEat Disord 48:535-554

MacLeod C, Mathews A (2012) Cognitive bias modification approaches to anxiety. Annu Rev Clin Psychol 8:189-217

Zilverstand A, Parvaz MA, Goldstein RZ (2017) Neuroimaging cognitive reappraisal in clinical populations to define neural targets for enhancing emotion regulation. A systematic review. Neuroimage 151:105-116

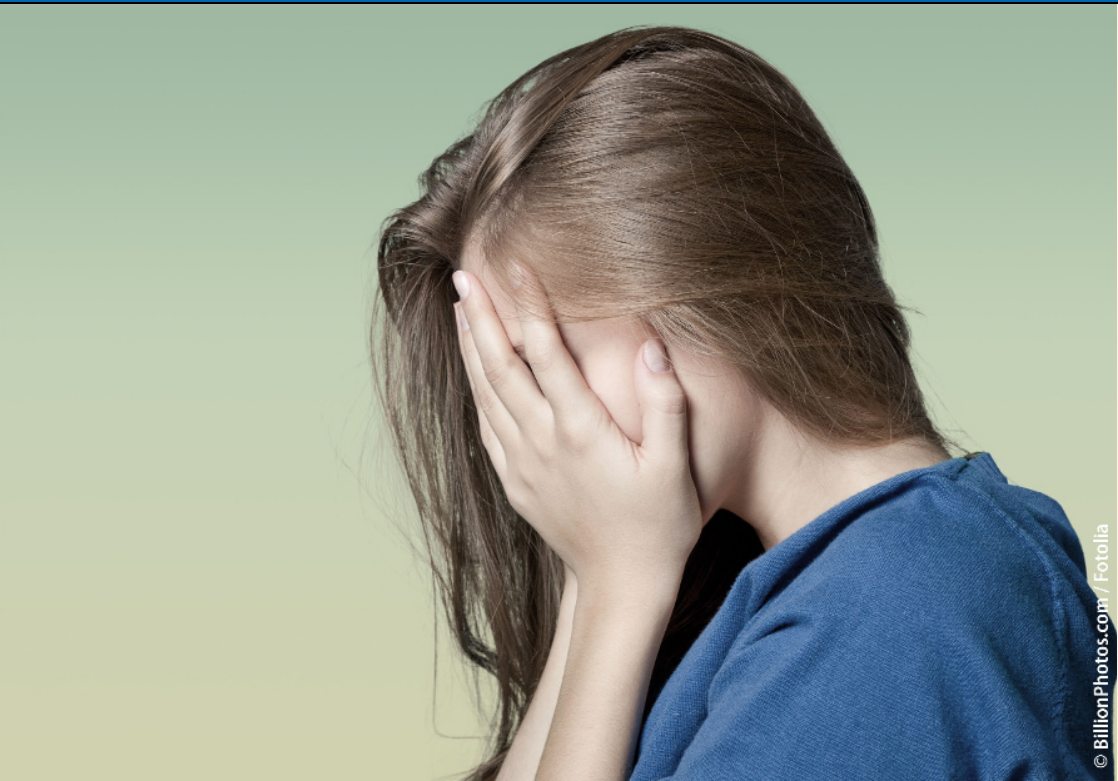

\section{Neuer Behandlungsansatz für Jugendliche und junge Erwachsene mit Gewalterfahrungen}

Sexueller und körperlicher Missbrauch an Kindern und Jugendlichen sind leider ein weit verbreitetes Phänomen - etwa 4 bis 16 $\%$ der Kinder erleben in westlichen Ländern körperliche Misshandlungen, bei sexuellem Missbrauch liegen diese Zahlen zwischen 5 und $10 \%$. Die Auswirkungen dieser negativen Kindheitserlebnisse sind vielfältig neben Einschränkungen in vielen Lebensbereichen haben Betroffene auch ein erhöhtes Risiko an einer psychischen Störung zu erkranken, insbesondere an der Posttraumatischen Belastungsstörung.

Bisher gab es nur für Kinder oder Erwachsene überprüfte Behandlungsverfahren - für die Altersgruppe dazwischen, Jugendliche und junge Erwachsene, existierten nur wenige Therapieansätze, deren Wirksamkeit in Studien bestätigt wurde.

Ein Team an der Katholischen Universität Eichstätt-Ingolstadt hat in den letzten Jahren daran gearbeitet, diese Lücke zu schließen. In einer großen, vom Bundesforschungsministerium geförderten Studie mit mehreren Behandlungsstandorten wurde eine speziell an die Bedürfnisse von Jugendlichen angepasste Therapie überprüft. Die Behandlung beruhte auf einem Ansatz der kognitiven Verhaltenstherapie mit 30 bis 36 Sitzungen verteilt über ein halbes Jahr, der sich bereits bei Erwachsenen bewährt hatte.
Es zeigte sich, dass die Gruppe, die die neue Therapie bekam, nach Behandlungsende deutlich weniger Symptome einer Posttraumatischen Belastungsstörung aufwies als die Kontrollgruppe. Aber auch andere psychische Erkrankungen, wie depressive Symptome oder Anzeichen einer BorderlinePersönlichkeitsstörung, gingen bei der behandelten Gruppe stärker zurück. Auch drei Monate nach Therapieende konnten diese Unterschiede beobachtet werden.

Die erfolgreiche Überprüfung der neuen Therapieform könnte nun zu einer Verbesserung der Behandlungssituation von traumatisierten Jugendlichen beitragen. Weitere Studien sollten nun untersuchen, ob und wie diese Therapie Einzug in die Versorgung finden kann.

\section{Originalpublikation:}

Rosner R, Rimane E, Frick U, et al. (2019) Effect of developmentally adapted cognitive processing therapy for youth with symptoms of posttraumatic stress disorder after childhood sexual and physical abuse: a randomized clinical trial. JAMA Psychiatry. doi:10.1001/jamapsychiatry.2018.4349

Quelle: Katholische Universität Eichstätt-Ingolstadt (http://www.ku.de) 